\title{
Residual stress measurement methods comparison in high-strength steel for naval application
}

\author{
LEVIEIL Bruno ${ }^{1,2 *}$, CALLOCH Sylvain ${ }^{1}$, DOUDARD Cédric ${ }^{1}$, \\ THEVENET David ${ }^{1}$, LEBLE Bruno ${ }^{2}$ \\ ${ }^{1}$ LBMS EA 4325 2, rue François VERNY 29806 BREST CEDEX 9 \\ ${ }^{2}$ DCNS Research 44620 LA MONTAGNE \\ *bruno.levieil@ensta-bretagne.fr
}

Keywords: Residual stresses, high-strength steel, contour method, X-Ray diffraction

\begin{abstract}
Residual stresses can be beneficial or detrimental to mechanical structures. In this work, a rectangular plate specimen with a cold expanded hole was designed to study the influence of these stresses on low cycle fatigue of high-strength steel. Two separated measurements of the stresses were made using different techniques: X-ray diffraction method and contour method. The results show good agreement with finite element modelling of the cold-expanded hole problem.
\end{abstract}

\section{Introduction}

Naval industry uses fabrication processes that induce a high-level of residual stresses in highstrength steel plates. A reliable fatigue life prediction requires thus the characterization of the residual stress influence on fatigue properties [1]. To investigate this influence, the use of particular experiment designs (geometry of the specimen, residual stress state, material, dynamic stress/strain level ...) is needed. Furthermore, it is important to be able to measure the initial residual stress and its evolution under fatigue loading in the considered design. In this work, a rectangular plate specimen with a circular hole is designed. A residual stress state is induced using cold-expansion technique: an oversized conical pin is passed through the hole causing yielding in the hole edge region. Schematic description of this process is presented in figure 1.

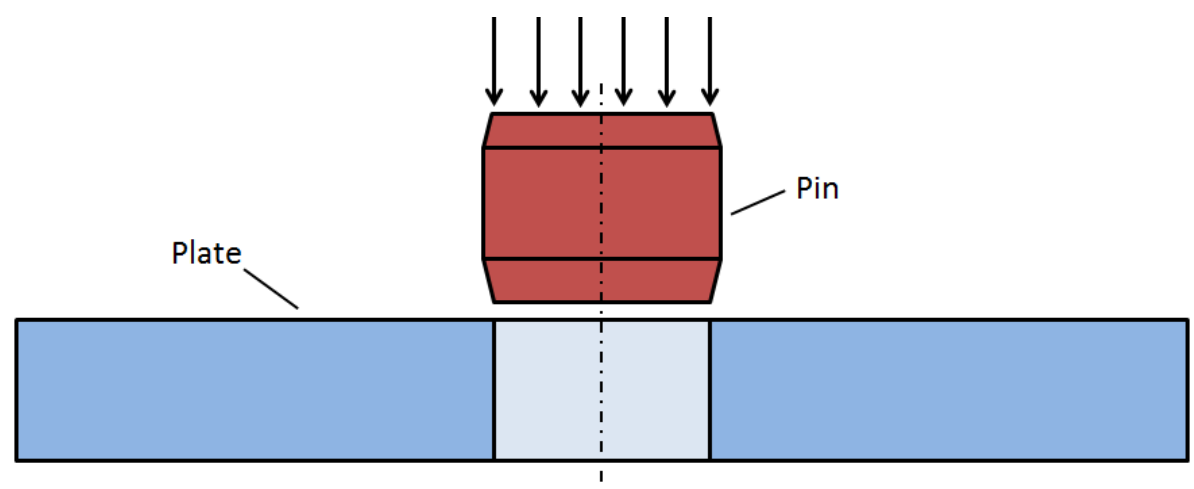

Fig. 1 : Introduction of residual stresses by cold expansion process.

The aim of this study is to compare different measurement techniques of residual stress for the studied case with finite element analysis (FEA) prediction. Experimental conditions will be described before to detail the numerical model used. This work is completed with two experimental evaluations of residual stresses: X-ray diffraction, and contour methods. 


\section{Experimental realization}

This work starts with the experiment description and the design precaution that have been taken to insure modelling hypothesis that will be presented later.

Geometry. Plates are cut by electric discharge machining (EDM) to guarantee initial free-stress state. Surface machining is completed by grinding to insure a "smooth" machining. An experimental assembly (see figure 2) is designed to control the geometry conditions. The entire top surface of the pin (5) is pushed through the hole of the guiding plate (4) which has a sliding fit with the pin so as to guarantee that the pin axis is collinear to the hole's axis of the specimen plate (3). A $100 \mathrm{kN}$ Tema traction-torsion machine has been used for this and for the material characterisation.

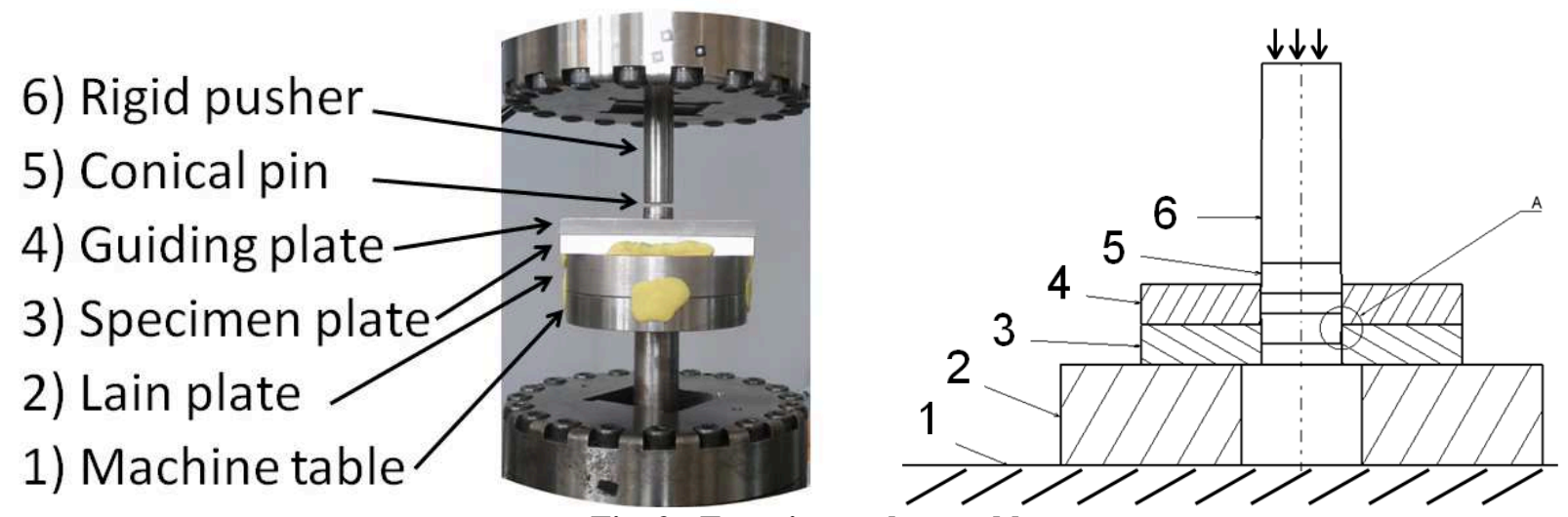

Fig. 2 : Experimental assembly

Material. The pin is made of hardened 35NCD16 steel to be sure that it remains elastic and can be used for several plates. Hardness Vickers testing was performed to guarantee its mechanical properties. The plate is made of high-strength steel used in naval industry. The material behaviour law (see table 1) includes negative and kinematic hardening which identification is achieved with the load-unload curve presented in figure 4 . This curve is built by loading a tensile specimen until yielding appears. Then, plastic deformation is added before reversing loading direction to obtain compression yielding. This cycle is repeated to get the hardening domain evolution.

Table 1 : Mechanical behaviour model equations

\begin{tabular}{|c|c|c|c|}
\hline $\begin{array}{c}\text { Total strain } \\
\text { separation }\end{array}$ & $\underline{\varepsilon}=\underline{\varepsilon}^{e}+\underline{\varepsilon}^{p}$ & Isotropic hardening $\mathrm{R}$ & $\dot{R}=b^{*}(Q-R) \dot{p}$ \\
\hline Hooke's law & $\underline{\varepsilon}^{e}=\frac{1}{E}\left[(1+v) \underline{\sigma}-v^{*} \operatorname{tr}(\underline{\sigma}) \underline{I}\right]$ & $\begin{array}{c}\text { Cumulated plastic } \\
\text { strain rate }\end{array}$ & $\dot{p}=\sqrt{\frac{2}{3} \underline{\varepsilon}^{p}: \dot{\varepsilon}^{p}}$ \\
\hline $\begin{array}{c}\text { Elasticity } \\
\text { domain f }\end{array}$ & $f=J_{2}\left(\underline{\sigma}-\underline{X}_{T}\right)-\sigma_{y}-R \leq 0$ & $\begin{array}{c}\text { Kinematic hardenings } \\
X_{\mathrm{i}} \text { summation }\end{array}$ & $\underline{X}_{T}=\sum \underline{X}_{i}$ \\
\hline $\begin{array}{c}\text { Plastic strain } \\
\text { rate }\end{array}$ & $\dot{\varepsilon^{p}}=\dot{p} \frac{3}{2} \frac{\underline{\sigma}^{d}-\underline{X}}{J_{2}(\underline{\sigma}-\underline{X})}$ & $\begin{array}{c}\text { Kinematic hardening } \\
X_{\mathrm{i}} \text { evolution }\end{array}$ & $\underline{X}_{i}=\frac{2}{3} C_{i} \dot{\varepsilon}^{p}-\gamma_{i} \underline{X}_{i} \dot{p}$ \\
\hline
\end{tabular}

The load-unload curve illustrates clearly the negative isotropic hardening $\mathrm{R}$ as domain size decreases while the stress-strain curve (figure 3) shows that the material model behaviour fits perfectly to the experimental data. In this paper, all the stresses are normalised with the same value so stress results can be compared. 


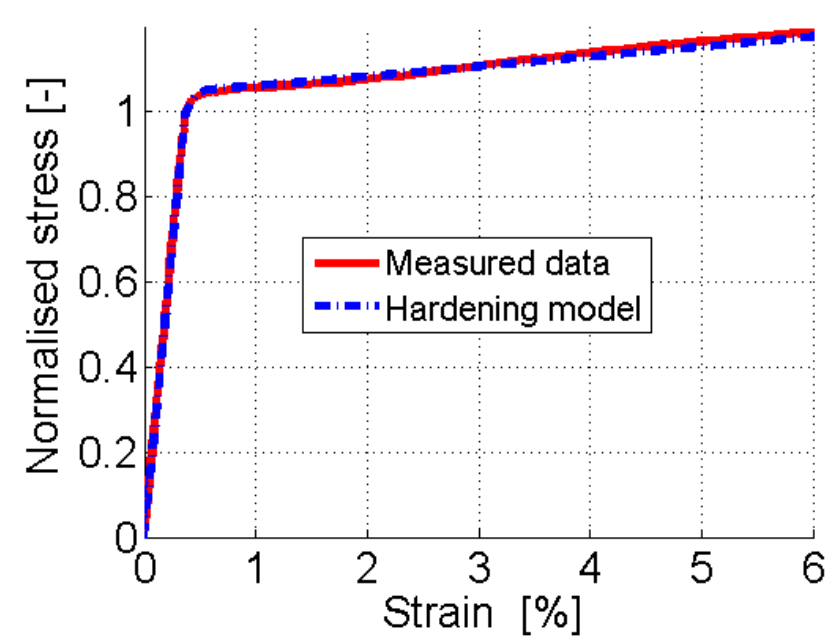

Fig. 3 : Normalised stress-strain curve

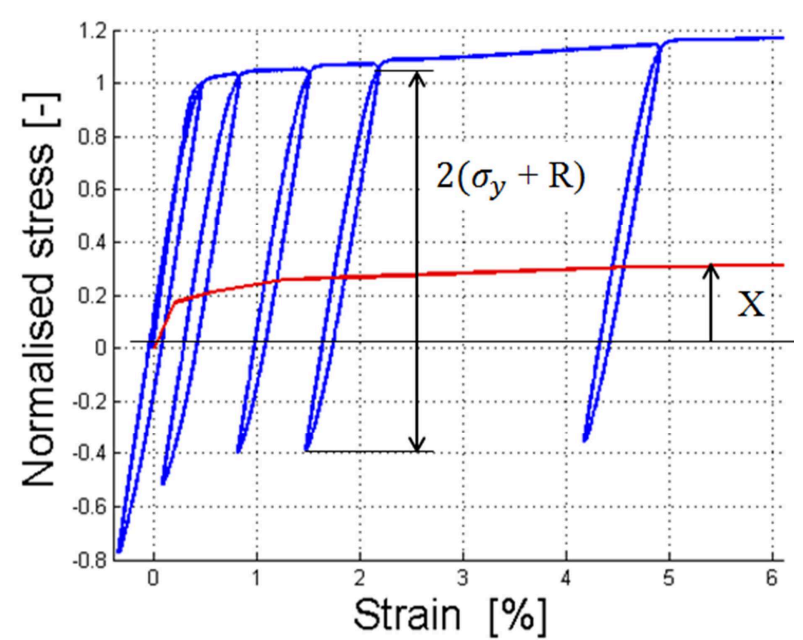

Fig. 4 : Normalised load-unload curve

\section{Finite element analysis}

Hypothesis. The model used here is a 3-D model representing one quarter of the problem (see figure 5). It considers the effect of the pin passing through the plate using a linear friction coefficient of 0.1 (classic value for non-lubricate steel/steel contact). A perfectly rigid surface pushes the pin through the plate which is maintained by an embedded rigid surface. 1400008 -node linear bricks with reduced integration and hourglass control were employed to mesh the parts.

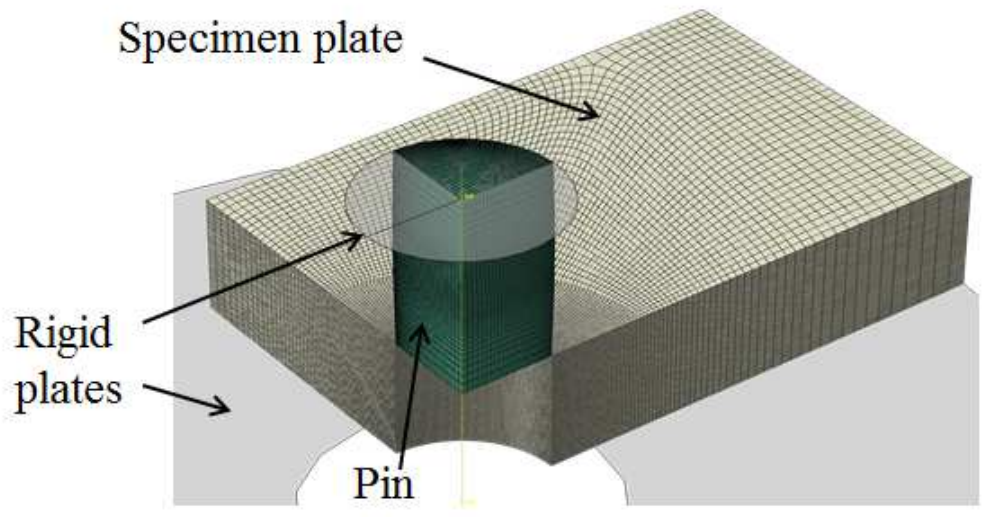

Fig. 5: 3-D modelling of the problem

Results. A study of the pin shape (conical, spherical...) and dimensions (diameter, cone angle) was carried out to design the one employed. No matter the pin design, the study showed that residual stresses distribution was heterogeneous across the thickness of the plate. Edges effects are observed and also an important drop in the residual stress value far from the edges. To homogenize stresses, surface machining is achieved. Figure 6 and 7 show the FEA residual stress state prediction in the plate before and after surface machining considering no efforts are induced by machining.
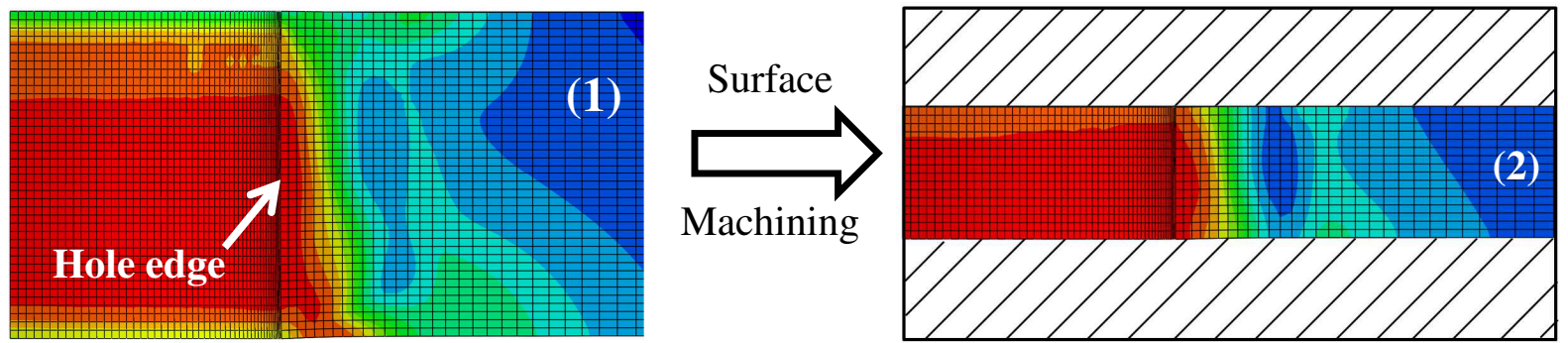

Fig. 6 : Von Mises stress states before (1) and after surface machining (2) 


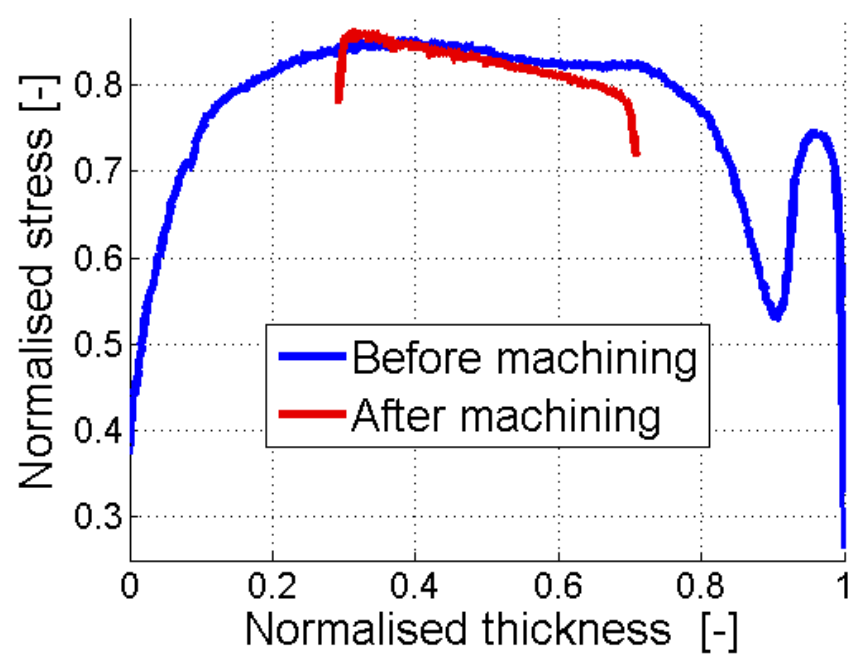

Fig. 7 : Residual Von Mises stress through the thickness before and after machining at the edge of the hole

\section{Residual stress measurements}

X-ray diffraction. XRD method was used first to evaluate the initial state of residual stresses due to the machining process. The complete method is described in several manuals such as [2]. A plate without induced residual stresses (i.e. in which no pin was passed through) was used as a reference state and X-Ray diffraction is performed on the top surface with a $2 \mathrm{~mm}$ spot. Results are shown in figure 8. It shows that, although significant residual stress state exists in the first $30 \mu \mathrm{m}$ of the thickness, it disappears afterward.

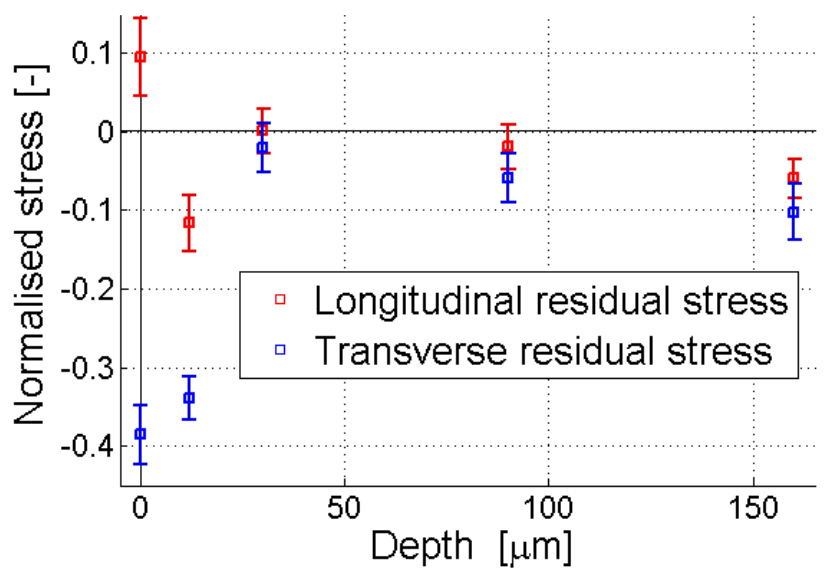

Fig. 8 : Stress state in the first $160 \mu \mathrm{m}$ of the thickness of a "stress-free" plate

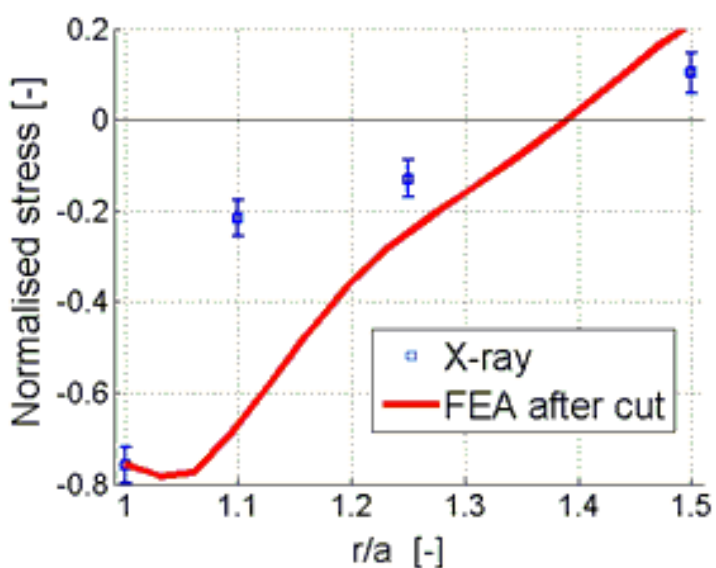

Fig. 9 : Comparison between FEA prediction of tangential residual stress after cut and XRD method

Measurements also indicate that important residual stresses exist at the edge of the hole. As these stresses disappear at a depth that is 10 times lower than the interpenetration of the pin in the radius of the hole plate, it is supposed that these stresses are relieved during the introduction of the pin and have no influence on the final results.

$\mathrm{X}$-ray residual stresses measurements results in a plate with pin-induced residual stresses are shown in figure 9 and compared to the FEA prediction of a plate cut in two equal halves. Reason is that XRay measurements in the radius of the hole needed the part to be cut for tool access. Cut is taken into account in the FEA by deleting the symmetry condition. 
Contour. The contour method was first developed by Prime [3] in 2001. It gives the complete 2D cartography of the normal residual stress on a whole surface that is previously cut. It has 3 steps: Cut, measure of the cut surface, post-treatment with FEA. Figure 11 illustrates the three stages involved in the contour method.
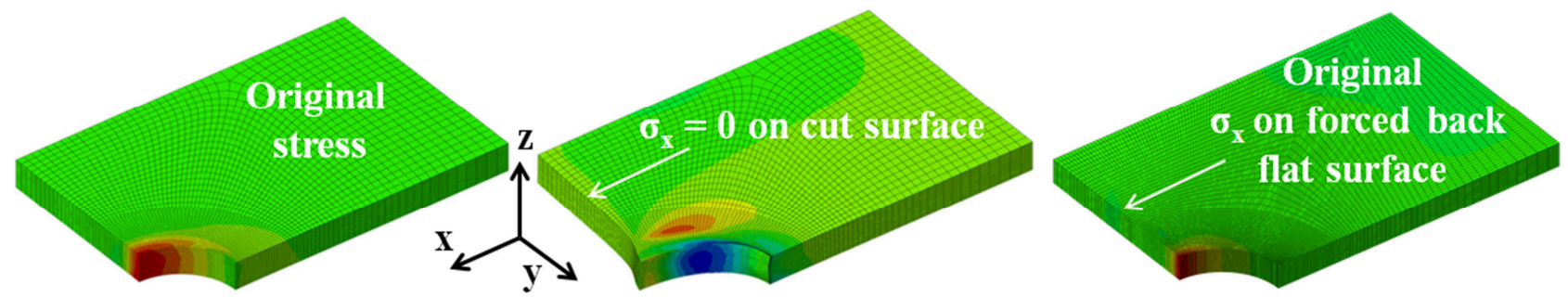

1) Original plate 2) Plate cut in 2 with relaxation with residual stresses of the free surface (strain $\mathrm{x} 100$ )

3)Stress-free deformed plate forced back

Fig. 10: Contour method illustration

The plate was cut in 2 symmetric halves by an EDM with an $\varnothing 0.1 \mathrm{~mm}$ wire. Appropriate clapping of the two sides of the plates was used to prevent relaxation while cutting. Surface topography was measured with a laser scanner GOM Atos with $10 \mu \mathrm{m}$ accuracy and averaged on the two halves to limit shear strain and EDM trajectory effect as detailed by Prime. Outliers were deleted but as data were noisy (due to laser scanning uncertainty), a fitted surface was used to smooth results. The fitted function was then introduced into a FEA software as boundary displacement condition to get the corresponding residual stress.

Comparison of the residual stress in the cut face normal direction (i.e. tangential residual stress in the hole coordinate system) is made with the FEA prediction in figure 12. Effect of pin friction can be seen as there is no horizontal symmetry plane (showing that $2 \mathrm{D}$ planar is a strong hypothesis) and the biggest differences are in the corners of the face, far from the hole.
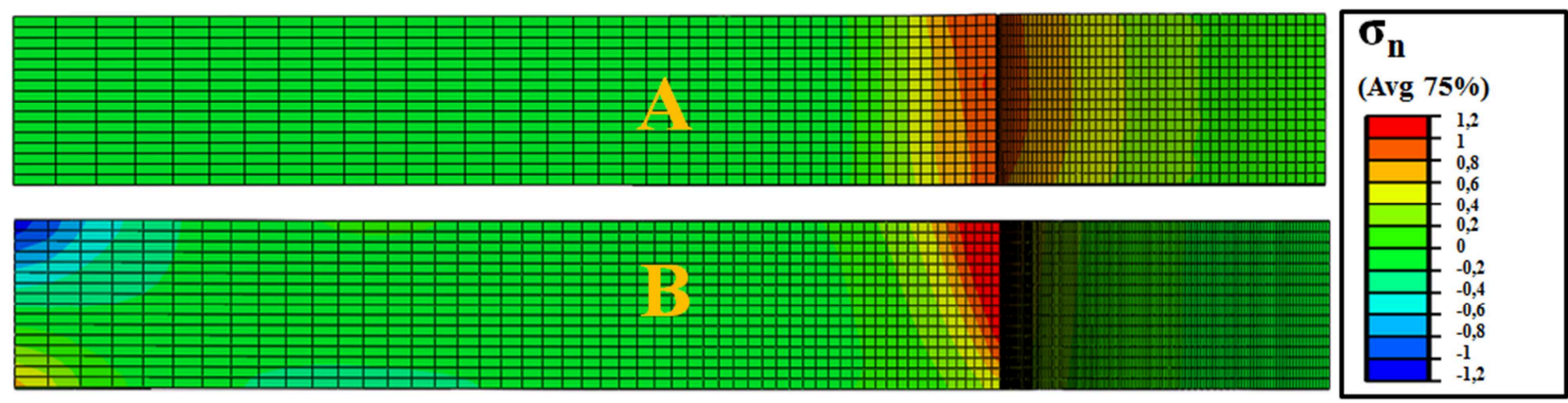

Fig. 9 : Normal stress comparison between FEA prediction (A) and contour method results (B)

Actually, when looking at tangential stress along the radius in the middle plane of the plate (figure 13), it can be observed that there is 3 zones where contour method results is far from FEA prediction: in $r=a, r=1.5 a$ and $r=4 a$. It corresponds to the zone where the fitted function doesn't represent well the data. Error is mostly due to the fitting function that could be optimised. 


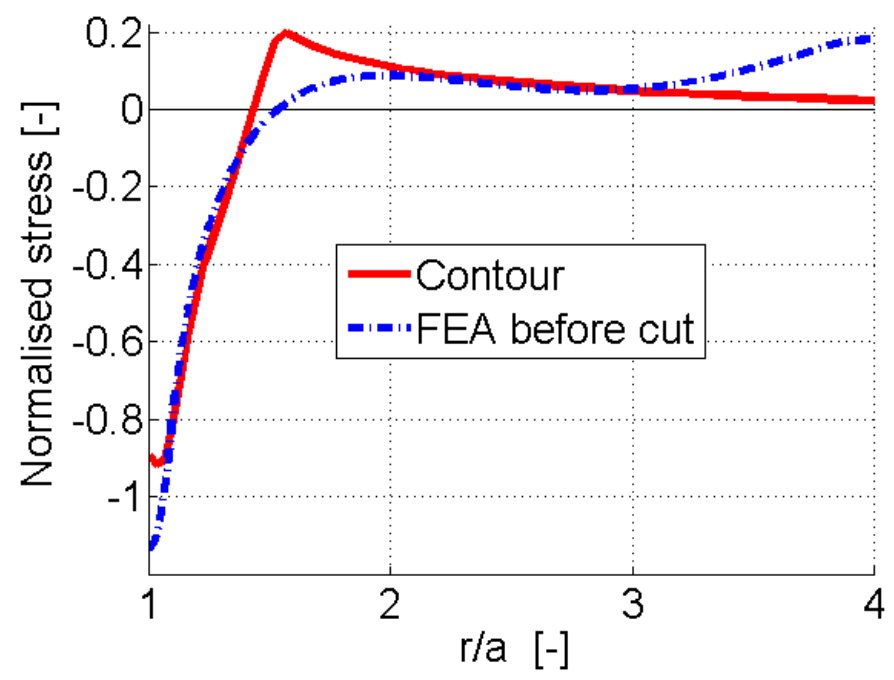

Fig. 10 : Comparison between FEA prediction of tangential residual stress and contour method measurements

\section{Conclusion}

Experimental study and FEA of the cold-expanded hole problem have been presented and residual stresses were evaluated with two different measurement techniques. Both X-Ray diffraction and contour methods showed good quantitative agreements with FEA predictions. These measurement techniques are complementary as they give different informations. Contour method allows getting the complete cartography of stresses in one direction whereas X-Ray diffraction gives discrete twodirectional information. Being able to ascertain FEA predictions is the first step that allows using the model for LCF study.

Two-dimensional FEA will serve for this purpose as it is faster than complete three-dimensional models. Residual stresses are introduced in the 2-D model by the application of a pressure at the edge of the hole. Correspondence between 2D (pressure applied around the hole) and 3D (pin passing through the hole) is done by calculating the pressure that if applied both to the pin and the hole edge gives a deformation equal to the interpenetration between pin and hole diameters.

In order to improve residual stresses accuracy, measurements of the relieved surface in the contour method should be made with a coordinate measuring machine.

\section{Acknowledgments}

The financial support of DGA/MRIS (Mission for Scientific Research and Innovation) is gratefully acknowledged.

\section{References}

[1] G.E. Totten Handbook of residual stresses and deformation of steel, ASM International, 2002

[2] G. S. Schäger, Practical residual stress measurement methods, Wiley 328 (2013) 139-161

[3] M.B. Prime, Cross-sectional mapping of residual stresses by measuring the surface contour after a cut, Journal of Eng. Mat. and Tech., 123 (2001) 162-168 